\title{
Genotyping of Digital Music Service Subscribers
}

\author{
Rafael R. Vargas \\ Deep Learning RD \\ info@deeplearningrd.com
}

September 2018

\begin{abstract}
This paper presents a methodology to categorize subscribers of digital music service (DMS) by taking as input variables their historic download pattern and streaming library. Drawing inspiration from biology, we develop a metric called genotype by defining a series of indicators called attractors and detractors that form a category space or species for every user. These species are based on four main styles of music: latin, urban, rock and pop; the indicators assign weights to the genres based on the sociological subjective perspective of music fans from one category in relation to other music styles, i.e., how they view other types of music they don't feel affinity with. The result is a segmentation of users that finds application in the making of offers and promotions, which can in turn be coupled with association rules and market basket analysis to improve direct marketing campaigns (CTR) and maximize revenue.
\end{abstract}

keywords: digital music, segmentation, association rules, genotype

\section{Introduction}

The digital music industry has grown exponentially in the last decade, fostered by the proliferation of smartphones, music applications and higher Internet bandwidth. The reduced cost of acquisition in comparison to hard-copy music and the ability to choose specific songs rather than buying whole albums are also determinant factors for this increasing trend. For companies offering music products, the challenge resides in how to optimize monetization of such products. Market basket analysis approaches have proven to be successful in the past by associating certain types of items (songs, albums, etc.) with others based on past consumption. The association can be done either vertically, i.e. looking at past purchases of the same user to offer them other songs from the same genre; or horizontally, by prompting the user to purchase songs based on other subscribers similar buying and liking patterns. 
The advent of music streaming has been the latest disruption of the music industry and now represents the vast majority of online subscriptions and purchases. Services like Spotify, Deezer and SoundCloud amass more than 200 million subscribers worldwide $^{1}$ and growing every day. Still, there are is a considerable amount of those user than don't subscribe to the services and use them as a free application.

The traditional method of monetizing non-paying users have been to add advertising between playing lists and embedded in the layout of the application. This freemium model has proven to be effective, offering basic service with in-app purchases with higher benefits to "popcorn effect" the way for paid subscriptions. We propose that, in addition to all these efforts, a deeper segmentation and genotyping of the user should be performed, to improve the changes of subscribing by offering something they would like to buy based on a specific categorization of his music style affinity.

\section{Association Rules and Market Basket Analy- sis}

In machine learning and data mining, Association Rule Learning is a technique for finding relations between several variables in a large database. The method was first proposed by Agrawal et $\mathrm{al}^{2}$ and takes to the surface deep relationships between items purchased or used by customers in a determined data space. For example, in the hardware store industry, if $A=$ wood, $B=$ nails and $C=$ hammer, the rule $\{A, B\} \Rightarrow C$ implies that customers who purchased woods and nails are likely to also buy a hammer with a likehood that depends on the support, confidence and lift ${ }^{3}$ of said rule.

The support of a rule is an indicator of purchase frequency. Formally, the support of $X$ with respect to $P$ is defined as the proportion of purchases $\mathrm{p}$ in the dataset $\mathrm{P}$ containing the itemset $\mathrm{X}$; and is given by:

$$
\operatorname{supp}(X)=\frac{|\{p \in P ; X \subseteq p\}|}{|P|}
$$

confidence is a value defining how often the rule has been found to be true and is given by:

$$
\operatorname{conf}(A \Rightarrow B)=\frac{\operatorname{supp}(A \cup B)}{\operatorname{supp}(A)}
$$

The lift of a rule is the proportion of observed support to the expected support value if item $\mathrm{A}$ and $\mathrm{B}$ were independent, or mathematically: 


$$
\operatorname{lift}(A \Rightarrow B)=\frac{\operatorname{supp}(A \cup B)}{\operatorname{supp}(A) \cap \operatorname{supp}(B)}
$$

There is also another useful metric called conviction which is the ratio of the expected frequency that $A$ happens without $B$ if they were independent, i.e. effectively the amount of error of the rule, divided by the total observed frequency of incorrect predictions made by the rule.

$$
\operatorname{conv}(A \Rightarrow B)=\frac{1-\operatorname{supp}(B)}{1-\operatorname{conf}(A \Rightarrow B)}
$$

The association rules are mined implementing the apriori algorithm, proposed by Agrawal and Srikant ${ }^{4}$. The pseudocode is as follows:

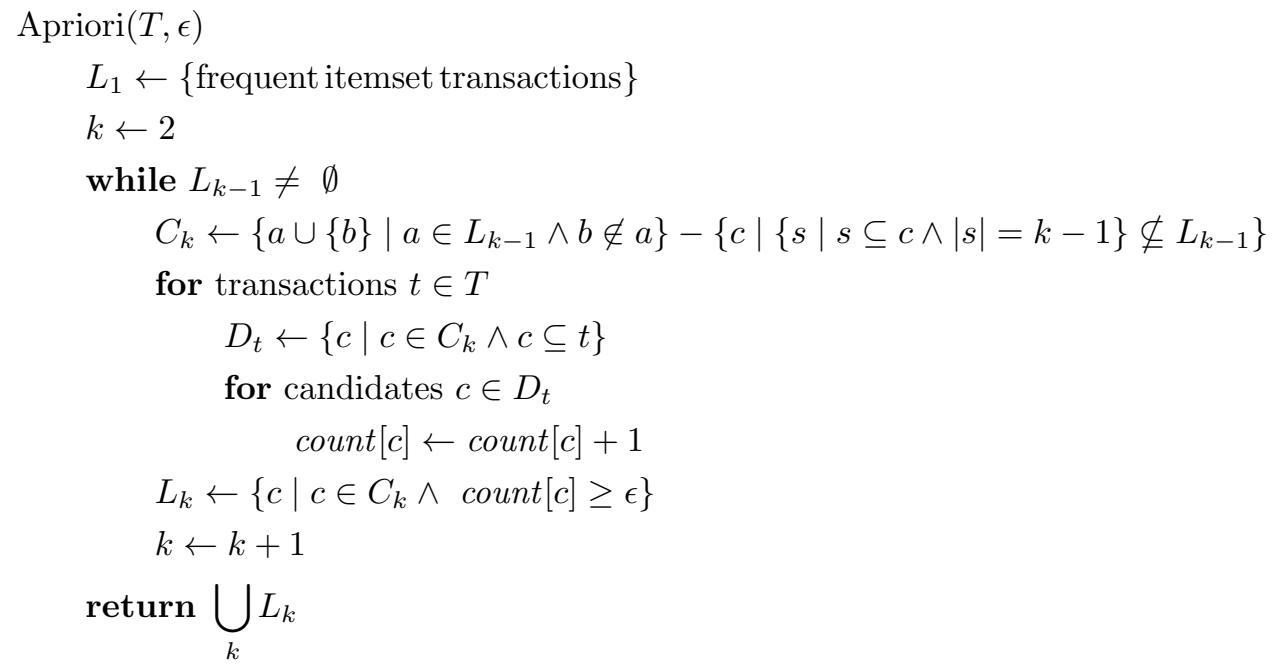

The algorithm is efficient for datasets containing timestamped transactions, such as the ones present in the digital music industry. There are other algorithms like WINEPI and MINEPI ${ }^{5}$ that can mine non-timestamped events; these fall under the category of episode rule mining. Apriori mines frequent itemsets and establishes the most common tuples between those itemsets, but it does not categorize the user performing the transaction.

We now introduce a method called the genotype, which addresses the user directly, rather than the itemsets in the data space. This metric can be used in tandem with traditional association rules to improve product placement and promotion effectiveness. 


\section{Subscriber Genotype Definition}

Genotyping is a type of user segmentation that aims to categorize users under different "species". These species are predefined by looking through the pattern of past purchases of each user and their historic CTR of specific promotions using $A / B$ testing $^{6}$. In our particular case we will limit the number of species (categories of users) to four, but $n$ number of species may be defined. Our four species will be:

- Latin Lovers: Users with a majority of latin related music in their historical profiles.

- Urbanizers: Users with a majority of urban related music in their historical profiles.

- Rock Evangelists: Users with a majority of rock related music in their historical profiles.

- Poppers 2.0: Users with a majority of pop related music in their historical profiles.

The genotyping of the species follows a combination of attractors and detractors which add or subtract weight to yield a total sum and a consequent classification. For this purpose, a species lookup matrix is constructed with all the coefficients for attractors and detractors of each musical style. This is represented in the following figure:

Fig. 1. Species lookup matrix

\begin{tabular}{|c|c|c|c|c|}
\hline Attractors & Urbanizer & Latin Lover & Popper 2.0 & Rock Evangelist \\
\hline $\mathrm{A} 1$ & $+3 R \& B$ & +3Merengue & +3Pop Dance & +3 Classic Rock \\
\hline $\mathrm{A} 2$ & +3 HipHop/Rap & +3Salsa & +3 Pop Rock & +3 Metal \\
\hline A3 & +1. 5Reggaeton & +2Reggaeton & +2Pop Rap & +2.5 Punk \\
\hline A4 & +1.5 Trap & +2 Bachata & +2Electronica & +2 Progressive Rock \\
\hline A5 & +1.5 Soul & +Mambo & +Trap & +Acoustic Rock \\
\hline A6 & +1.5Pop Rap & +Power Ballads & +Power Ballads & +0.5Electronica \\
\hline \multicolumn{5}{|l|}{ Detractors } \\
\hline D1 & -3 Metal & -3HipHop/Rap & -3 Metal & -3Reggaeton \\
\hline D2 & -3 Punk & -3 Metal & -3Progressive Rock & -3HipHop/Rap \\
\hline D3 & -2Classic Rock & -2.5 Punk & -2Classic Rock & -2 Trap \\
\hline D4 & -1.5 Pop & -2Progressive Rocl & -2 Punk & -1.5 Merengue \\
\hline D5 & -1.5Electronica & -Pop - English & -HipHop/Rap & $-1.5 \mathrm{Salsa}$ \\
\hline D6 & -Salsa & -0.5 Electronica & -Merengue & -Bachata \\
\hline
\end{tabular}

As shown in Fig.1., each species has their own attractors and detractors coming from the perception each type of user have of the other music genres. The coefficients for each attractor/detractor represent the specific weight each of the music styles have in defining a given species. For example, a Latin Lover would have high attraction weights for Latin type of music like Salsa and Mambo, but low or no attraction (detraction) for Rock or Pop. 


\title{
4 Algorithm Breakdown
}

The idea of genotyping is to provide a categorization of users into musical genres. The datasets used to mine these categorizations must at least have fields for customerID, downloaded or streamed songs, classification of the songs into different musical styles and timestamp of the download or streamed action done by the user. Also, as mentioned before, a species lookup matrix must be constructed for referencing the songs and associate them with the attractors and detractors coefficients in order to perform the necessary calculations that will output the categorization value

The general formula for the genotype of a user over the transactions made in his service subscription tenure has the form:

$$
\max \left\langle\sum\left(\prod_{i \in I} X_{i}\right)\right\rangle
$$

where $\prod_{i \in I} X_{i}$ is the cartesian product of the number of songs with all the species coefficients:

$$
\prod_{i \in I} X_{i}=\left\{f: I \rightarrow \bigcup_{i \in I} X_{i} \mid(\forall i)\left(f(i) \in X_{i}\right)\right\}
$$

The following is a pseudocode for the implementation of genotyping for the entire user history of downloads or streamed music:

\author{
begin method genotype \\ input vars $\leftarrow$ customerID, download $\|$ streamed history, songStyleTag \\ initialize species lookup matrix[ ] \\ for customerID $=1$ to $\mathrm{n}$ do \\ search download || streamed history \\ get songStyleTag \\ match songStyleTag with lookup matrix coefficients \\ for each songStyleTag do \\ cartesian product of matrix coefficients with nb of songs \\ end do \\ sum values grouped by songStyleTag \\ output max(sum values grouped by songStyleTag) \\ end do
}




\section{Application to a sample dataset}

We have constructed a fictitious sample dataset based on subscriptions to a digital music service to test our method. The dataset is composed of 72,727 unique users with a history of downloaded songs properly categorized into different musical genres. The song genres were pseudo-randomly distributed using the Wichman-Hill ${ }^{7}$ seed generator. We used MS Excel and R library $x l s x$ to read and format the data frames and perform the calculations. ggplot2 and Excel built-in chart options were used for visualization and graphics. The data set is available at www.musimathician.com/documents ${ }^{3}$

Fig. 2. Visualization of user genotyping

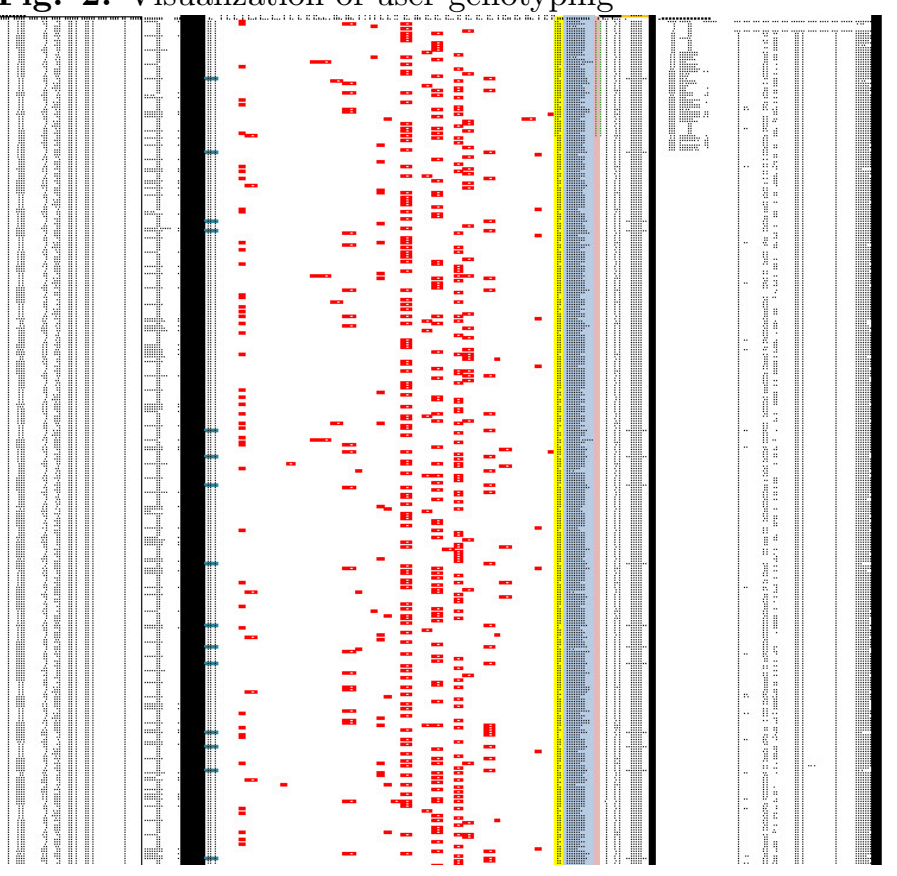

Fig. 3. Screenshot of genotyping segmentation

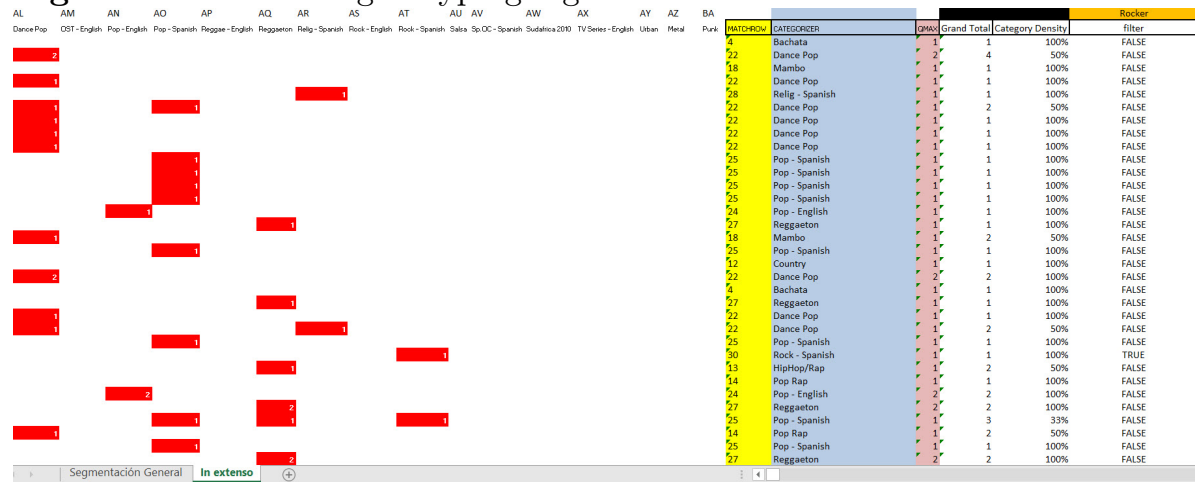




\section{$6 \quad$ Results and findings}

After applying the genotyping methodology to our dataset we found the following general segmentation of the subscriber base.

Fig. 4. General segmentation of subscriber base

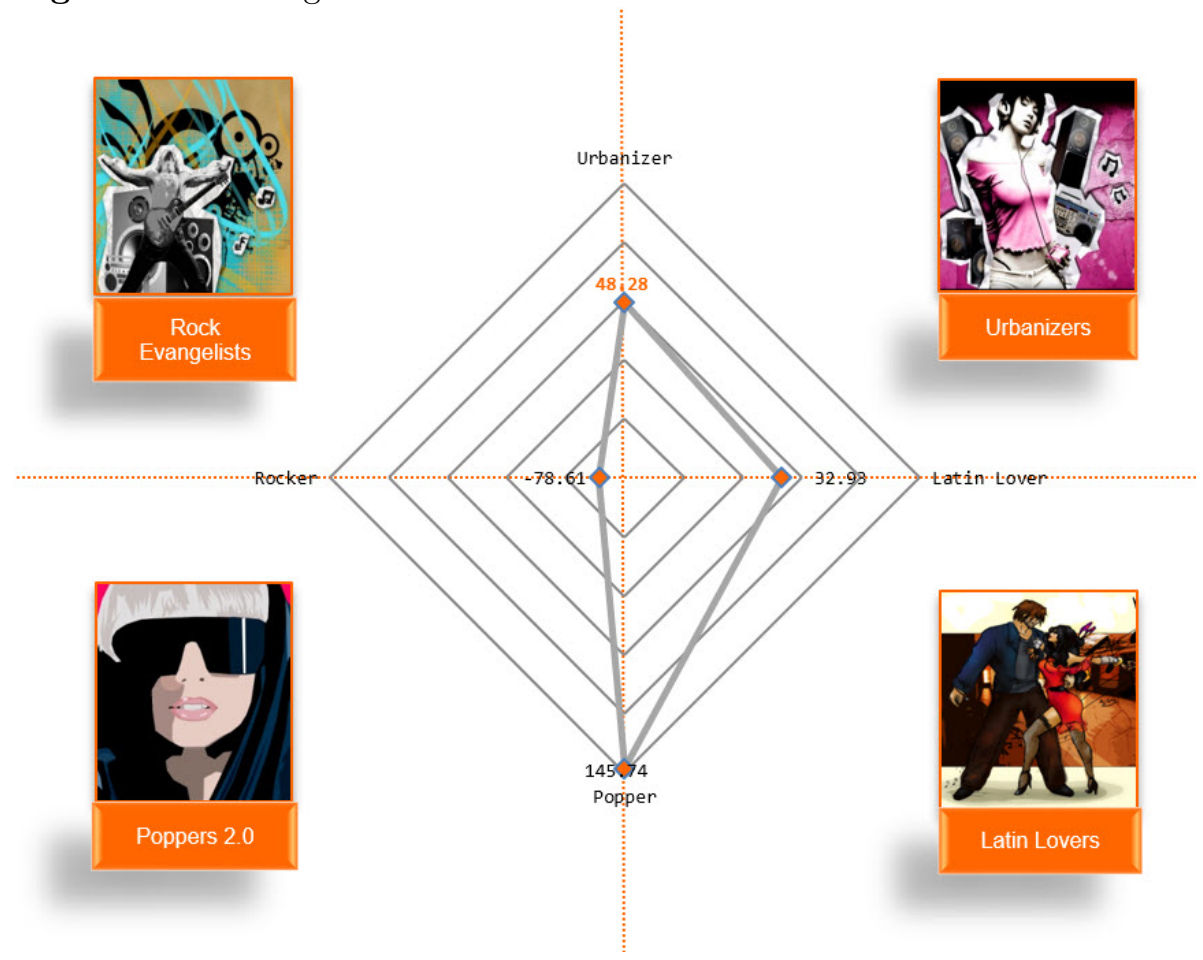

The base lean towards the Poppers segment, meaning they are the main species within the dataset, urbanizers being a distant second. After the establishment of the species, each user is effectively categorized within a group and can then be targeted with usage-based promotions and pricing offers based on their preferred musical styles. Proactive advertising campaigns can be deployed for each segmented group to maximize CTR; also, horizontal recommendations can be made using associations rules mined from other members of the same species. 


\subsection{Limitations}

\subsubsection{Hybridization or neutrality}

It could be the case that some users do not show a particular segmentation because their genotype is evenly mixed and the weights all balance out. For these cases, the recommended approach is to narrow the search for download or streamed history to the last four weeks of transactions to account for the nearest to current day behavior of the user. This can be generalized by adding a trend factor or exponential moving average factor with a high $\alpha$ to add more weight to recent events.

\subsubsection{Asymmetry in song distribution}

Genotyping assumes that there is an even distribution of songs across musical genres in the database, but this may not be the case in a practical scenario where the universe of available songs may lean towards a particular musical style. This could introduce bias in the segmentation, since it's obvious that as there are more songs of a particular genre there would be more downloads of said genre. This can be addressed by normalizing the data with a smoothing factor that balances the contributions of each genre proportionally to the entire database.

\section{Conclusions}

In this paper we have discussed a novel way of segmenting users of digital music services by looking through their historical data of downloaded or streamed songs. We defined the genotype method with four species of users and provided example code for its implementation. We tested the method in a ad-hoc dataset to watch the results and discussed about the possibilities of its use in conjunction with association rules and market basket analysis to improve marketing campaigns and maximize revenue.

We also called attention to segmentation bias due to an uneven distributions of songs in the database and to the possibility of no-classifying a user due to a balanced weight score when testing for the species. We then proceeded to give specific recommendations on how to deal with those issues. 


\section{References}

[1]"Spotify Technology S.A. Announces Financial Results for Second Quarter 2018". Spotify Investors. 26 July 2018. Retrieved 26 July 2018.

[2] Agrawal, R.; Imieliski, T.; Swami, A. Mining association rules between sets of items in large databases. Proceedings of the 1993 ACM SIGMOD international conference on Management of data - SIGMOD '93. p. 207. 1993

[3] Hahsler, M. Introduction to arules A computational environment for mining association rules and frequent item sets. Journal of Statistical Software. 2005

[4] Agrawal, R. and Srikant, R. Fast algorithms for mining association rules. Proceedings of the 20th International Conference on Very Large Data Bases, VLDB, pages 487-499, Santiago, Chile, September 1994.

[5] Guillame-Bert, M.; Crowley, J.L. Learning Temporal Association Rules on Symbolic Time Sequences. Asian Conference on Machine Learning. JMLR: Workshop and Conference Proceedings 25:159174. 2012

[6] Kohavi, R.; Longbotham, R. Online Controlled Experiments and A/B Tests. In Sammut, Claude; Webb, Geoff. Encyclopedia of Machine Learning and Data Mining. 2017, Springer.

[7] Wichmann, B. A.; Hill, I. D. Algorithm AS 183: An Efficient and Portable Pseudo-Random Number Generator. Journal of the Royal Statistical Society. Series C (Applied Statistics). 31 (2): 188190.

[8] Vargas, R. Genotype Segmentation dataset. www.musimathician.com/documents 